\title{
Texture-induced magnetic interactions in ferrofluids
}

\author{
A. Urtizberea, ${ }^{1,2}$ A. Arizaga,,${ }^{1,2}$ N. J. O. Silva, ${ }^{3}$ A. Millán, ${ }^{1,2}$ F. Palacio, ${ }^{1,2}$ and F. Luis, ${ }^{1,2, a)}$ \\ ${ }^{1}$ Instituto de Ciencia de Materiales de Aragón (ICMA), Universidad de Zaragoza - CSIC, Facultad de \\ Ciencias, Pedro Cerbuna 12, 50009 Zaragoza, Spain \\ ${ }^{2}$ Departamento de Física de la Materia Condensada, Universidad de Zaragoza, Facultad de Ciencias, \\ Pedro Cerbuna 12, 50009 Zaragoza, Spain \\ ${ }^{3}$ Departamento de Física and CICECO, Universidade de Aveiro, 3810-193 Aveiro, Portugal
}

(Received 25 October 2011; accepted 29 March 2012; published online 4 May 2012)

\begin{abstract}
We report a method for reversibly controlling the strength of dipole-dipole interactions in maghemite ferrofluids. In order to induce some magnetic texture, the ferrofluid is exposed to a strong magnetic field while it is cooled from room temperature to below its freezing temperature. The experimental data show that the average strength of dipolar interactions increases with increasing texture and that the magnetic relaxation becomes slower. (c) 2012 American Institute of Physics. [http://dx.doi.org/10.1063/1.4709725]
\end{abstract}

\section{INTRODUCTION}

The properties of interacting magnetic nanoparticles have been extensively studied in the last few decades. ${ }^{1}$ Interactions are relevant for technological applications as they affect the data stability and switching magnetic fields in high density granular ${ }^{2}$ and patterned ${ }^{3}$ magnetic media. From a more fundamental point of view, dipolar interactions lead to a plethora of complex magnetic phenomena, not present in the case of individual nanomagnets. Open scientific questions, which are still subject of considerable debate, are the existence of a (super)spin-glass phase at sufficiently low temperatures ${ }^{4-7}$ and the influence that dipolar interactions have on the superparamagnetic relaxation time..$^{8-12}$

Experimental studies of these questions usually rely on the modification of sample parameters which control the strength of dipolar interactions, such as (1) the concentration of nanoparticles in frozen ferrofluids, ${ }^{4,5,13}$ granular composites, ${ }^{14}$ or polymers, ${ }^{15,16}$ and (2) the average particle size ${ }^{17}$ and the number of layers ${ }^{18}$ in self-organized granular multilayers. However, these studies face some technical difficulties. In solutions, it is usually difficult to avoid the tendency of magnetic nanoparticles to aggregate. In multilayered materials, by contrast, this effect can be minimized, as the spatial arrangement of nanoparticles is approximately preserved. Yet, in this case, there are also some unavoidable uncertainties in parameters such as the particle size and its distribution, which might slightly vary between different samples prepared by the same method.

In the present work, we report on an experimental method that enables us to "switch on" and "off" dipolar interactions. In a ferrofluid, the easy axes orientations can be controlled, to some extent, via the application of a sufficiently strong magnetic field in the liquid state. ${ }^{19-21}$ Within this approach, neither the number of nearest neighbours nor the distribution of particle sizes change. Therefore, differences between the magnetic relaxation times measured in the randomly oriented and textured materials can be safely associated with changes in the strength of dipolar interactions.

a)Electronic mail: fluis@unizar.es.
In the case of very diluted ferrofluids, with negligible dipolar interactions, the texture process does not induce by itself an increase of the blocking temperature. ${ }^{21}$ In the present work, by contrast, we used much more concentrated ferrofluids. Because of their intrinsic anisotropic character, dipolar interactions depend on the relative orientations of the magnetic anisotropy axes. The texture process increases the strength of dipolar interaction, enabling us the study of the influence of dipolar interaction on the magnetic relaxation.

The paper is organized as follows. In Sec. II, we describe the experimental methods employed to synthesize and characterize the ferrofluids. The results of magnetic measurements are reported in Sec. III. They provide a detailed information on the magnetic texture attained by the application of a magnetic field as well as on the pronounced influence that texture has on magnetic dipole-dipole interactions and relaxation rates. Section IV is left for the conclusions.

\section{EXPERIMENTAL METHODS}

\section{A. Synthesis of ferrofluids}

In this paper, we study a ferrofluid of maghemite nanoparticles dispersed in dioctyl ether. The synthesis of highly crystalline and monodisperse maghemite nanocrystallites was carried out in organic medium following the Hyeon method. ${ }^{22}$ This procedure, which allows varying the particle size by controlling the amount of surfactant, is based on the thermal decomposition of iron pentacarbonyl in the presence of oleic acid. The resulting iron nanoparticles were transformed into monodisperse maghemite by a controlled oxidation using trimethylamine oxide as a mild oxidant. All the reagents were purchased from Aldrich.

In a typical synthesis of $10 \mathrm{~nm}$ diameter nanoparticles, $0.4 \mathrm{ml}$ of $\mathrm{Fe}(\mathrm{CO})_{5}$ were injected into a mixture containing $20 \mathrm{ml}$ of octyl ether and $2.56 \mathrm{~g}$ of oleic acid at $100^{\circ} \mathrm{C}$ under a constant argon flow. The resulting mixture was heated to reflux and maintained at this temperature $\left(280^{\circ} \mathrm{C}\right)$ for $1 \mathrm{~h}$. During this process, the initial yellow colour of the solution gradually turned into black, indicating the formation of iron nanoparticles. The resulting black solution was cooled down 
to room temperature and $0.34 \mathrm{~g}$ of $\left(\mathrm{CH}_{3}\right)_{3} \mathrm{NO} \cdot 2 \mathrm{H}_{2} \mathrm{O}$ were added. The mixture was heated up to $130{ }^{\circ} \mathrm{C}$ under argon atmosphere and maintained at this temperature for $2 \mathrm{~h}$. Then, it was slowly heated to reflux, maintained at this temperature for $1 \mathrm{~h}$, and cooled down to room temperature. For the magnetic experiments, the original ferrofluid was diluted to a concentration of $0.88 \mathrm{mg} \mathrm{Fe}_{2} \mathrm{O}_{3} / \mathrm{ml}$.

\section{B. Crystallographic characterisation}

The X-ray powder diffraction (XRD) pattern of the dried sample (not shown) agrees with an inverse spinel structure, thus suggesting the formation of either magnetite or maghemite. The $d$ spacing and the intensities predicted for these two phases are, however, very similar. It is therefore impossible to differentiate them by XRD due to the peak broadening associated with the nanoscopic size of the crystalline domains. The results of a titration analysis proved the absence of Fe (II), thus showing that the samples are made of pure maghemite $\left(\gamma-\mathrm{Fe}_{2} \mathrm{O}_{3}\right)$.

\section{Morphology and particle dispersion}

The size distribution and morphology of the particles were studied by means of transmission electron microscopy (TEM) and dynamic light scattering (DLS). A representative image of the nanoparticles in the ferrofluid is shown in Fig. 1. The nanoparticles present a spherical shape with average diameter $D=8 \pm 1 \mathrm{~nm}$. The analysis of DLS data (not shown) provides an average hydrodynamic diameter of about $10 \mathrm{~nm}$. This value is slightly higher than $D$ due to the oleic acid layer surrounding each nanoparticle, appreciable also in the TEM image. These results confirm that nanoparticles do not aggregate to form clusters at room temperature.

Small angle x-ray scattering (SAXS) was measured in order to analyze the influence that a $H=10 \mathrm{kOe}$ magnetic field has on the nanoparticle arrangement in the ferrofluid (notice that $M$ is very close to saturation $M_{\mathrm{s}}$ at RT and $10 \mathrm{kOe}$ ). The experiments were performed at the Beam Line BM16 of the European

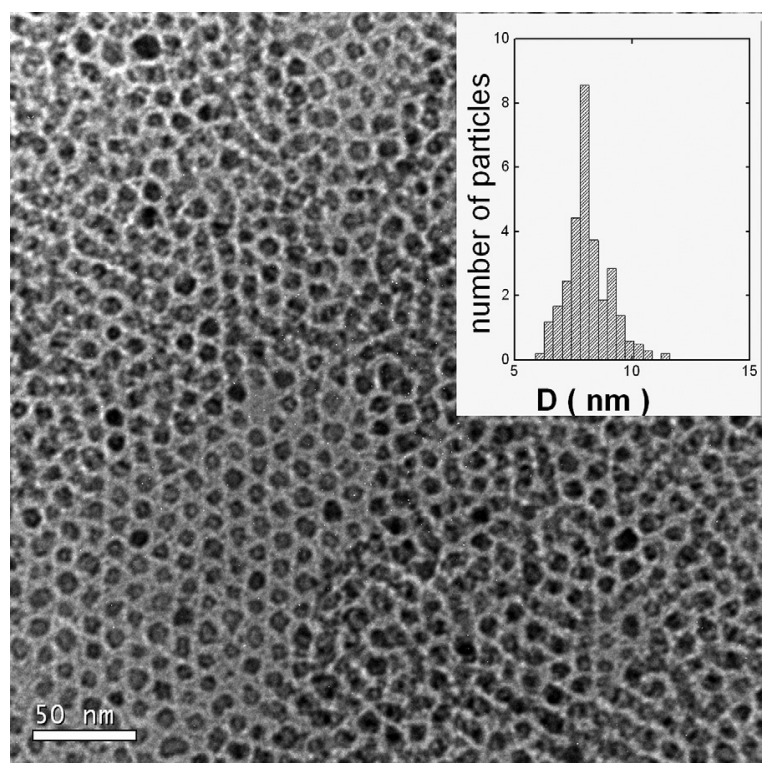

FIG. 1. TEM image of the $\gamma-\mathrm{Fe}_{2} \mathrm{O}_{3}$ ferrofluid. Inset: Distribution of particle diameters determined from the analysis of TEM data.
Synchrotron Radiation Facility (ESRF). The intensity profiles measured at room temperature and at $H=0$ and $H=10 \mathrm{kOe}$ are shown in Fig. 2. The fact that the intensity profiles are constant at low- $q$ suggests that there is no aggregation in the ferrofluids, as observed in the DLS measurements. In addition, the scattering profile measured at $H=10 \mathrm{kOe}$ agrees perfectly with that obtained at $H=0$. These results indicate that the magnetic field does not induce any aggregation at room temperature. A fit of these scattering profiles using a model of polydisperse solid spheres was made with GNOM software. ${ }^{23}$ The fit provides an average diameter of about $8.5 \mathrm{~nm}$, in agreement with the one determined by TEM.

\section{Magnetic measurements}

Magnetic measurements were performed with a Superconducting Quantum Interference Device (SQUID) magnetometer (MPMS-XL, Quantum Design) in the temperature range between 1.8 and $325 \mathrm{~K}$ and under magnetic fields up to $50 \mathrm{kOe}$. The ac susceptibility was measured by applying an oscillating magnetic field. The frequency $\omega / 2 \pi$ of the ac magnetic field was varied between 0.5 and $852 \mathrm{~Hz}$ and its amplitude was $h_{0}=4 \mathrm{Oe}$.

\section{RESULTS AND DISCUSSION}

\section{A. Control of magnetic texture}

In order to control the magnetic texture of the sample, two different cooling protocols were performed. They are described in Fig. 3. In the first one, here after designed as protocol 1, the sample was cooled to the lowest temperature $(1.8 \mathrm{~K})$ under no magnetic field (random sample, RDM).

After this process, the easy axes of the nanoparticles are oriented at random. The fraction of particles with the easy axes making an angle $\psi( \pm \mathrm{d} \psi)$ with the measuring field is $P(\psi) \sin \psi \mathrm{d} \psi$, where the orientational distribution of easy axes $P(\psi)=1$ for $0 \leq \psi \leq \pi / 2$. In the second one, designed

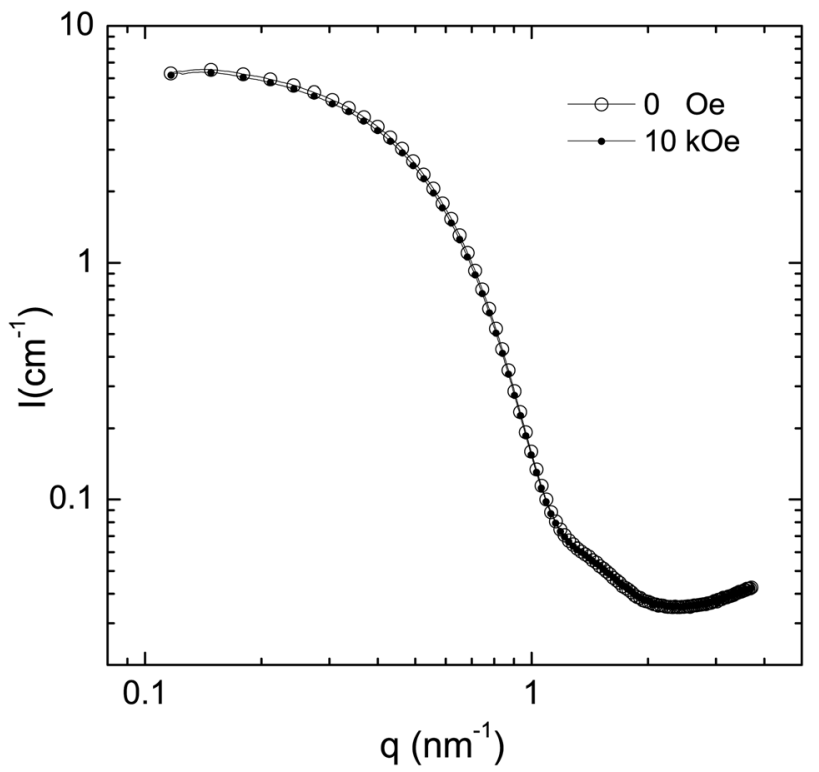

FIG. 2. SAXS intensity profiles measured at room temperature. Solid symbols represent the scattering profile in the presence of a magnetic field $H=10 \mathrm{kOe}$. The SAXS intensity in the absence of any magnetic field is also shown (open symbols). 

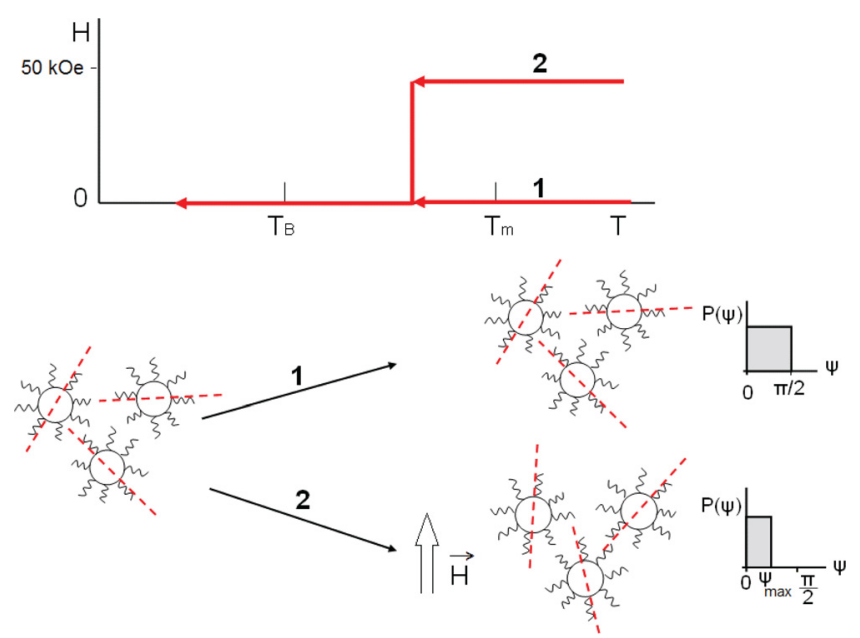

FIG. 3. Experimental protocols employed to control the magnetic texture. Protocol 1: The sample is cooled down from room temperature to $1.8 \mathrm{~K}$ in zero magnetic field; the easy axes of the nanoparticles stay oriented at random. Protocol 2: The sample is frozen to $110 \mathrm{~K}$ under a magnetic field of 50 $\mathrm{kOe}$; it is subsequently cooled to $1.8 \mathrm{~K}$ (crossing $T_{\mathrm{B}}$ ) in zero field. The angle $\psi$ that the easy axes made with the measuring field ranges from zero to $\psi_{\max }$, which is zero for a perfect alignment and $\pi / 2$ for randomly oriented axes.

as protocol 2, the sample was cooled from 280 to $110 \mathrm{~K}$, i.e., crossing the melting point of the solvent $\left(T_{\mathrm{m}} \sim 250 \mathrm{~K}\right)$, under a strong magnetic field of $50 \mathrm{kOe}$ (textured sample, TXT). Then, the sample was subsequently cooled from $110 \mathrm{~K}$ to the lowest temperature under no magnetic field. Notice that, since $T_{\mathrm{m}} \gg T_{\mathrm{B}}$ (the highest blocking temperature $T_{\mathrm{B}}$ is about $100 \mathrm{~K}$, see below) both protocols were zero field cooled (ZFC) processes with respect to the supermagnetic blocking.

After protocol 2, the easy axes of the nanoparticles are expected to rotate towards the texturing field, parallel to the measuring field. We model this effect with a narrower distribution function

$$
P(\psi)=\left\{\begin{array}{l}
1, \quad 0 \leq \psi \leq \psi_{\max } \\
0, \quad \psi \geq \psi_{\max }
\end{array}\right.
$$

where $\psi$ can have any value between zero and $\psi_{\max }$. For a perfect alignment of the easy axes $\psi_{\max }=0$. As a first approximation, we consider that $\psi$ is homogeneously distributed between zero and $\psi_{\max }$ (see Fig. 3 ).

The effect of the cooling protocol on the magnetic texture can be asserted by the properties, remanence, and coercivity of the hysteresis loops measured at $T \ll T_{\mathrm{B}}$. The area of the hysteresis loop at $T=2 \mathrm{~K}$, shown in Fig. 4, is larger for sample TXT than for sample RDM. This feature suggests that protocol 2 introduces a partial alignment of the easy axes with respect to the direction of the freezing field. In agreement with this, the remanent magnetization $M_{\mathrm{r}}$ increases slightly, by about $15 \%$, after protocol 2 . The degree of magnetic texture was determined from the remanent magnetization, which can be written as ${ }^{24}$

$$
M_{\mathrm{r}} \simeq\langle\cos \psi\rangle M_{\mathrm{S}}
$$

where $M_{\mathrm{s}}$ is the saturation magnetization and $\langle\cos \psi\rangle$ is given by

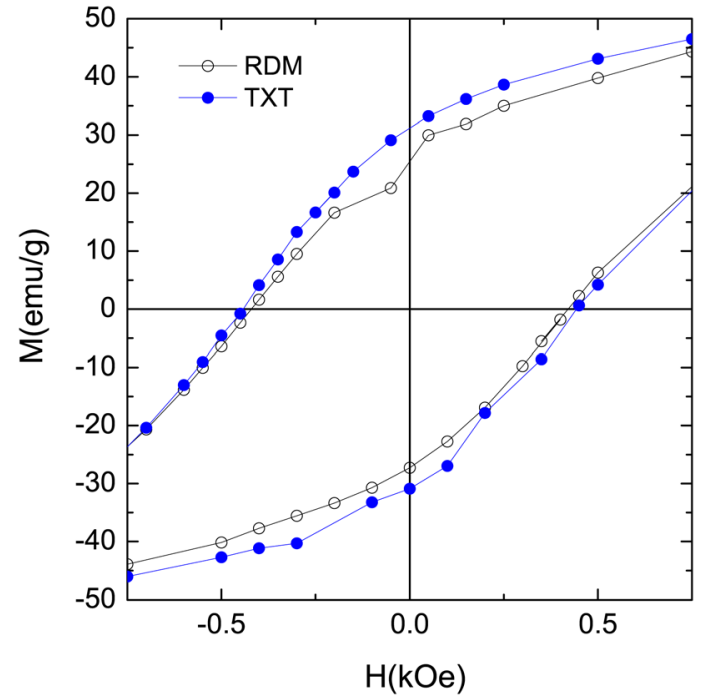

FIG. 4. Hysteresis loop of the RDM (०) and TXT (•) samples at $T=2 \mathrm{~K}$. The area increases after the protocol 2.

$$
\langle\cos \psi\rangle=\frac{\int_{0}^{\pi / 2} \cos \psi P(\psi) \sin \psi \mathrm{d} \psi}{\int_{0}^{\pi / 2} \sin \psi P(\psi) \mathrm{d} \psi} \simeq \frac{1-\cos 2 \psi_{\max }}{4\left(1-\cos \psi_{\max }\right)} .
$$

Inserting in Eq. (2) $M_{\mathrm{r}}$ and $M_{\mathrm{s}}$ of sample RDM, we obtain $\left\langle\cos \psi_{\mathrm{RDM}}\right\rangle=0.45$. This value is slightly smaller than that expected at $T=0$ for a sample with the easy axis oriented at random, $\langle\cos \psi\rangle=0.5$, since the hysteresis loops were measured at $T=2 \mathrm{~K}$, and Eq. (2) is strictly valid only in the limit of $T \rightarrow 0$. In order to avoid this experimental difficulty, $\left\langle\cos \psi_{\mathrm{TXT}}\right\rangle$ was determined from the ratio between the remanent magnetization of sample TXT and that of sample RDM. Considering that saturation magnetization of sample TXT is the same as that of sample RDM, one can write

$$
\frac{M_{r \mathrm{RDM}}}{M_{\mathrm{rTXT}}}=\frac{\left\langle\cos \psi_{\mathrm{RDM}}\right\rangle}{\left\langle\cos \psi_{\mathrm{TXT}}\right\rangle} .
$$

Inserting in Eq. (4) the experimental values for remanent magnetizations, $M_{\mathrm{rRDM}}$ and $M_{\mathrm{rTXT}}$, and using $\left\langle\cos \psi_{\mathrm{RDM}}\right\rangle$ $=0.5$ gives $\left\langle\cos \psi_{\mathrm{TXT}}\right\rangle=0.575 \pm 0.005$, which corresponds to $\psi_{\max }=81 \pm 1^{\circ}$.

The changes induced in the magnetic texture by the cooling protocol affect also the equilibrium susceptibility $\chi_{T} \cdot{ }^{25}$ As Fig. 5 shows, the in-phase susceptibility of sample TXT is larger than that of sample RDM above the blocking temperature but below the melting point. Interestingly, the susceptibilities of both samples merge above the melting point of the solvent, indicating that the texturing process is reversible. In principle, one can use these effects to determine the magnetic texture. However, $\chi_{T}$ is also influenced by the dipolar interactions (see Sec. III B below).

In order to avoid this difficulty, the degree of magnetic texture was estimated from the zero-temperature limit of the in-phase susceptibility. At $T \rightarrow 0$,

$$
\chi^{\prime} \simeq\left\langle\sin ^{2} \psi\right\rangle \chi_{\perp},
$$

where 


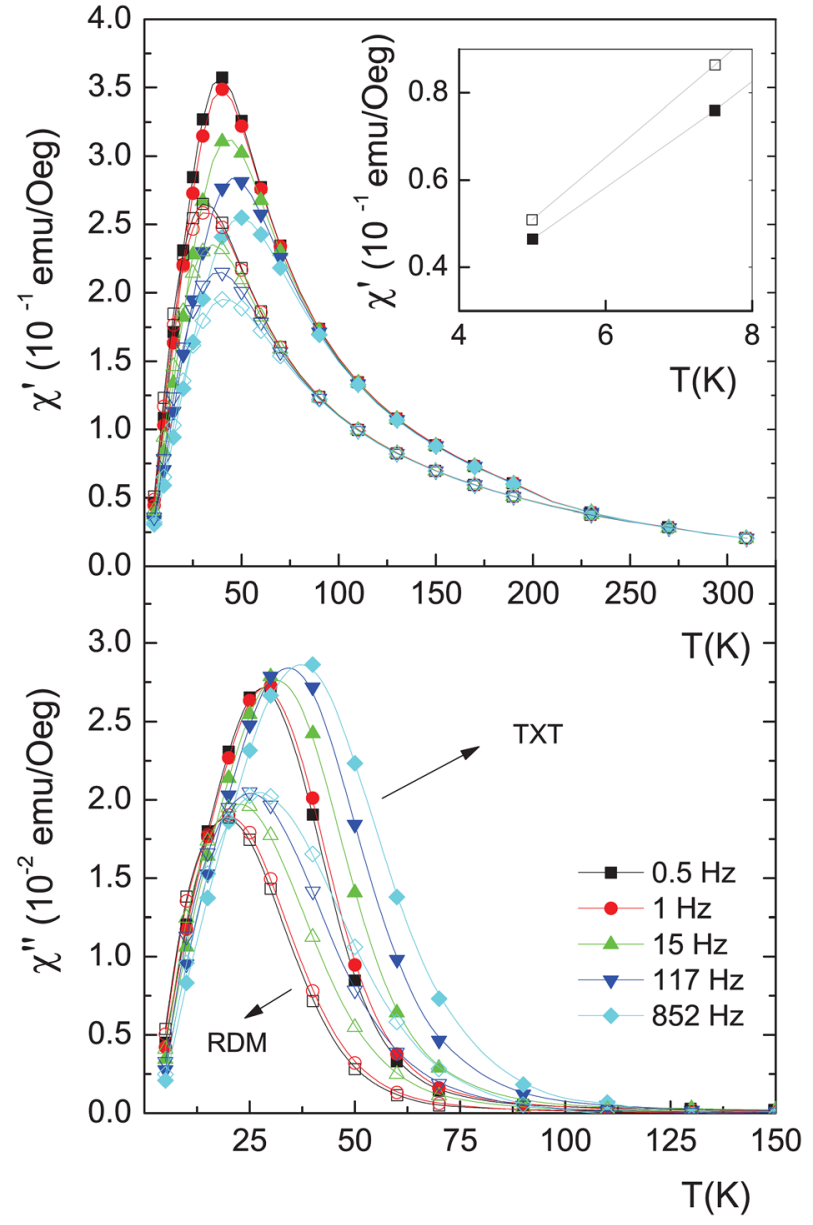

FIG. 5. AC susceptibility curves measured at different frequencies: Solid symbols are for the TXT; open symbols are for the non textured sample (RDM). Above the melting point of the solvent $T_{m} \simeq 250 \mathrm{~K}$ the susceptibility of sample TXT agrees with that of sample RDM. Inset: Magnification of the low temperature region

$$
\left\langle\sin ^{2} \psi\right\rangle=\frac{\int_{0}^{\pi / 2} \sin ^{3} \psi P(\psi) \mathrm{d} \psi}{\int_{0}^{\pi / 2} \sin \psi P(\psi) \mathrm{d} \psi} \simeq \frac{2+\cos ^{3} \psi_{\max }-\cos \psi_{\max }}{3\left(1-\cos \psi_{\max }\right)} .
$$

$\chi_{\perp}=M_{\mathrm{S}} / H_{\mathrm{AN}}$ is the equilibrium susceptibility perpendicular to the easy axis, rather insensitive to interactions, and $H_{\mathrm{AN}}$ the anisotropy field. The value $\chi_{\perp} \simeq 7.64 \times 10^{-12} \mathrm{emu} / \mathrm{Oe} \mathrm{g}$ was estimated using the in-phase susceptibility of sample RDM and assuming that in this sample the easy axes are randomly oriented, i.e., that $\left\langle\sin ^{2} \psi_{\mathrm{RDM}}\right\rangle=2 / 3$. For sample TXT, the same Eq. (5) gives then $\left\langle\sin ^{2} \psi_{\mathrm{TXT}}\right\rangle=0.608 \pm 0.001$, or $\psi_{\max }=81.2 \pm 0.2^{\circ}$, in excellent agreement with the value determined from the remanent magnetization.

The degree of texture obtained from remanence and susceptibility data can be compared with the theoretical values calculated in Ref. 24. According to this theory, the average value of $\cos \psi$ in a fluid dispersion of nanoparticles with magnetic moment $\mu$ and anisotropy energy $K V$, exposed to a magnetic field $H$, is given by

$$
\langle\cos \psi\rangle \simeq \mathcal{L}(\beta)\left(1-\frac{1}{2 \sigma}\right),
$$

where $\sigma=K V / \mathrm{k}_{\mathrm{B}} T, \beta=\mu H / \mathrm{k}_{\mathrm{B}} T$, and $\mathcal{L}(\beta)$ is the Langevin function. On cooling the sample through its freezing point $T_{\mathrm{m}}$, it retains the texture characteristic of the fluid at $T_{\mathrm{m}}$ characterized by $\beta=\mu H / \mathrm{k}_{\mathrm{B}} T_{\mathrm{m}}$ and $\sigma=K V / \mathrm{k}_{\mathrm{B}} T_{\mathrm{m}}$. The anisotropy energy $K V=485 \mathrm{~K}$ was determined from the out-of-phase susceptibility data of sample RDM following the method described in Ref. 26. The average magnetic moment at $T_{\mathrm{m}}, \mu=7.42 \times 10^{3} \mu_{\mathrm{B}}$ was obtained from the inphase susceptibility data of sample RDM. Inserting these values in Eq. (7) gives $\langle\cos \psi\rangle \simeq 0.73$, which corresponds to $\psi_{\max }=63^{\circ}$. The degree of texture expected from these calculations is, therefore, larger than the one obtained experimentally. The magnetic texture in a fluid dispersion of nanoparticles depends on the coupling between the magnetic moment and the easy axis of each particle. The discrepancy between the calculated and the experimental degrees of texture can be explained considering a weaker anisotropy, probably due to the fact that the anisotropy constant $K$ decreases with increasing temperature. Also, this theory does not take into account the fluid viscosity, which hinders the rotation of the magnetic particle within the carrier liquid. In spite of this quantitative discrepancy, we can safely conclude, on basis of the remanence and susceptibility data, that the ferrofluid becomes magnetically textured after protocol 2 .

\section{B. Dipolar interactions}

We next discuss whether the texture has any influence on the strength of the interparticle magnetic interactions. Figure 6 shows the reciprocal susceptibilities $1 / \chi^{\prime}$ of RDM and TXT samples. A simple way to model the effect of interactions is to assume that the equilibrium susceptibility $\chi_{T}$ follows the Curie-Weiss law

$$
\chi_{T} \simeq\left\langle\cos ^{2} \psi\right\rangle \chi_{\|}=\left\langle\cos ^{2} \psi\right\rangle \frac{C_{\|}}{k_{\mathrm{B}}(T-\theta)},
$$

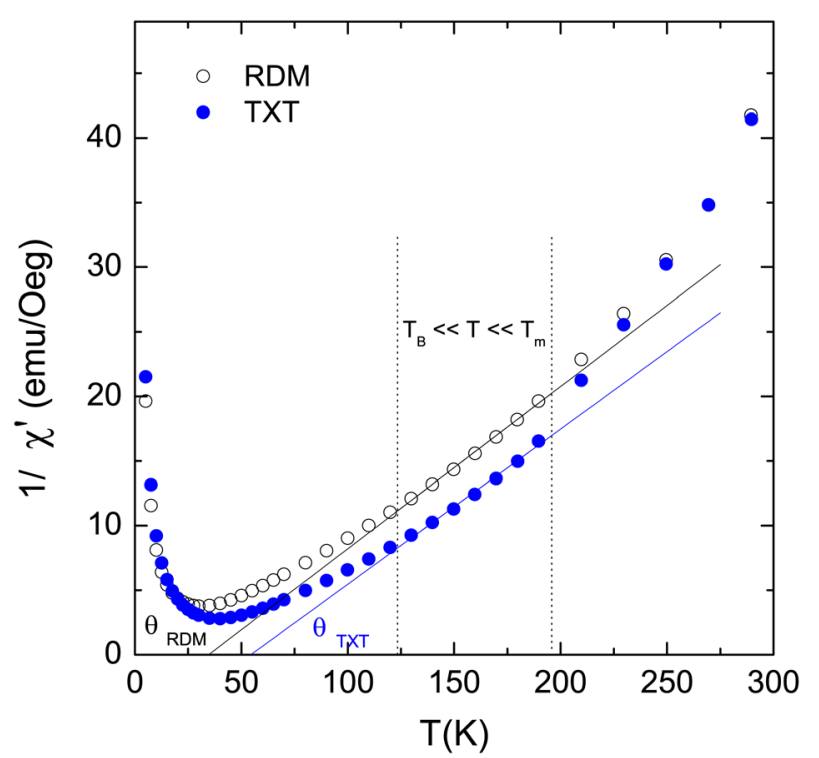

FIG. 6. Reciprocal ac susceptibilities of the TXT (solid symbols) and the RDM (open symbols) samples. The solid lines are Curie-Weiss fits of data measured in the temperature region $124 \mathrm{~K} \leq T \leq 196 \mathrm{~K}$, in which the susceptibilities of both samples attain their thermal equilibrium values. 
where $\chi_{\|}$is the susceptibility along the anisotropy axis, $C_{\|}=N \mu^{2}, N$ is the number of nanoparticles per gram and the Weiss temperature $\theta$ reflects the strength of interactions. ${ }^{27} \theta$ is usually obtained from the extrapolation of the reciprocal susceptibility to 0 . However, a linear dependence of $1 / \chi_{T}$ with temperature is not clearly observed (see Fig. 6), deviations being more noticeable as $T$ approaches $T_{\mathrm{B}} .{ }^{27}$ For this reason, the Curie-Weiss fit was made for temperatures well above $T_{\mathrm{B}}$ but below $T_{\mathrm{m}}$. The fits give $\theta_{\mathrm{RDM}}$ $=34.6 \pm 2.6 \mathrm{~K}$ and $\theta_{\mathrm{TXT}}=54.4 \pm 3.6 \mathrm{~K}$ for samples $\mathrm{RDM}$ and TXT, respectively (see Fig. 6). The increase of $\theta$ observed after cooling the ferrofluid in a magnetic field (protocol 2) supports the interpretation that the average interaction strength increases with increasing the magnetic texture.

This result can be understood considering the anisotropic character of dipolar interactions. Protocol 2 introduces a partial alignment of the easy axes along the magnetic field, which leads to a narrower distribution $P(\psi)$. The orientation of the magnetic moment of each particle becomes, in average, closer to that of its neighbours, thereby increasing the strength of dipolar interactions. We can conclude that the texturing process is able to enhance the magnitude of the dipolar interactions by orienting the easy axes of the nanoparticles.

\section{Magnetic relaxation: Influence of magnetic texture and interactions}

We can now attempt to explore how the texture and the magnetic interactions induced by it modify the magnetic relaxation process. In the absence of dipolar interactions, the ac susceptibility of a set of independent magnetic nanoparticles is ${ }^{28}$

$$
\chi \simeq\left\langle\cos ^{2} \psi\right\rangle \chi_{\|} \frac{1}{1+i \omega \tau}+\left\langle\sin ^{2} \psi\right\rangle \chi_{\perp},
$$

where \langle\rangle is the average over the distribution of easy axes orientations (see Sec. III A). The in-phase and out-of-phase susceptibility components are then given by ${ }^{28}$

$$
\begin{gathered}
\chi^{\prime}=\left\langle\cos ^{2} \psi\right\rangle \chi_{\|} \frac{1}{1+(\omega \tau)^{2}}+\left\langle\sin ^{2} \psi\right\rangle \chi_{\perp}, \\
\chi^{\prime \prime}=\left\langle\cos ^{2} \psi\right\rangle \chi_{\|} \frac{(\omega \tau)}{1+(\omega \tau)^{2}} .
\end{gathered}
$$

Notice from Eq. (11) that after the texture process, one may expect that $\chi^{\prime}$ and $\chi^{\prime \prime}$ components increase because $\left\langle\cos ^{2} \psi\right\rangle$ increases.

The relaxation time $\tau$ corresponding to the relaxation of the particle magnetic moment over the effective energy barrier $U_{\text {eff }}$ is given, at $H=0$, by the Néel-Brown expression ${ }^{29-31}$

$$
\tau=\tau_{0} \exp \left(U_{\text {eff }} / k_{\mathrm{B}} T\right),
$$

where $\tau_{0}$ is the attempt time and $U_{\text {eff }}$ has contributions resulting mainly from the magnetic anisotropy. The superparamagnetic blocking temperature $T_{\mathrm{B}}$, defined as the temperature of maximum $\chi^{\prime \prime}$ for a given frequency $\nu$, corre- sponds approximately to the condition $\nu=1 / 2 \pi \tau$. Notice that $\tau$ does not depend on the orientation of the easy axis at $H=0$. This argument applies also to systems with a distribution of particle sizes and therefore of relaxation times. Then, the texture process does not change by itself the magnitude of $\tau$. By contrast, $\tau$ and thus also $T_{\mathrm{B}}$ can be greatly influenced by the presence of dipolar interactions. ${ }^{8-12}$ In this case, $U_{\text {eff }}$ has additional contributions resulting from the dipole-dipole interactions. ${ }^{26}$ It might, therefore, depend on the degree of magnetic texture, only if the latter affects the interaction.

The susceptibility data displayed in Fig. 5 show that the out-of-phase susceptibility increases after the texture process, which indicates a larger susceptibility component along the anisotropy axis for sample TXT as compared to sample RDM. This effect arises, in part, from the texture of the easy axes along the direction of the texturing field (see Eq. (11)) and from the interaction effects discussed above (see Sec. III B).

The blocking temperatures for $\nu=0.5 \mathrm{~Hz}$ are $T_{\mathrm{B}} \sim$ $19.4 \mathrm{~K}$ and $T_{\mathrm{B}} \sim 28.3 \mathrm{~K}$ for samples RDM and TXT, respectively. These data suggest that relaxation has become much slower in sample TXT. The dependence of the relaxation time on the blocking temperature $T_{\mathrm{B}}$ of samples RDM and TXT is shown in Fig. 7. It follows the Arrhenius law ${ }^{29-31}$

$$
\ln (\tau)=\ln \left(\tau_{0}\right)+\frac{U_{\text {eff }}}{k_{\mathrm{B}} T_{\mathrm{B}}} .
$$

Figure 7 shows that $\tau$ of sample TXT is larger than $\tau$ of sample RDM at any temperature. Since the magnetic texture does not modify by itself $\tau$, as it is discussed above, the large effect observed must be associated with the stronger interactions present in the textured sample. The energy barrier $U_{\text {eff }}$ determined from the Arrhenius fits is also larger for sample TXT, $U_{\text {effTXT }}=893 \mathrm{~K}$, than for sample RDM, $U_{\text {effRDM }}=485 \mathrm{~K}$. In addition, $\tau_{0}$ of sample TXT is three orders of magnitude

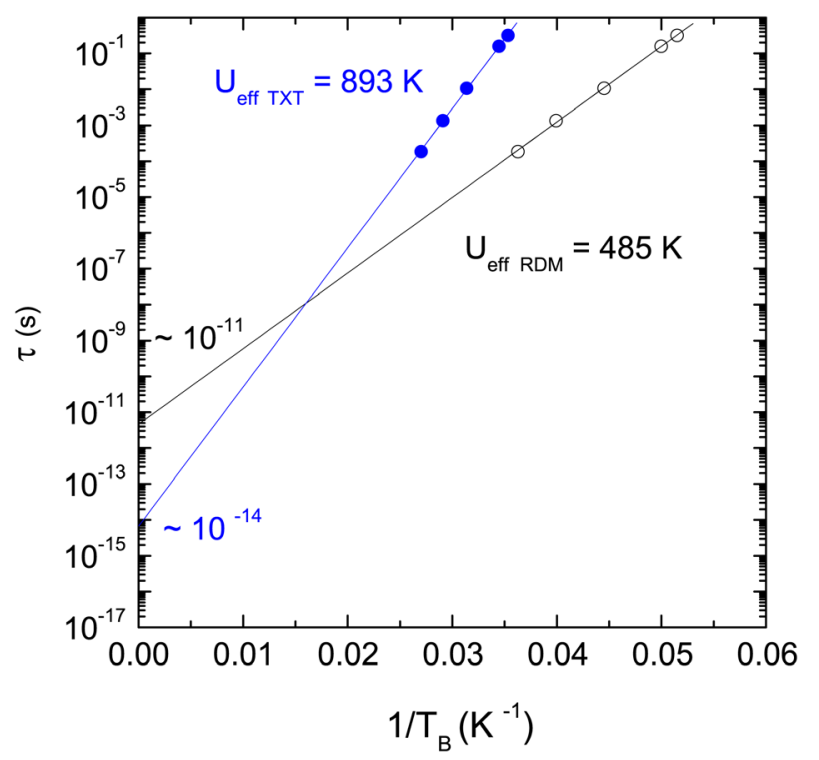

FIG. 7. Arrhenius plot of the magnetic relaxation time of RDM (O) and TXT samples $(\bullet)$. The values of the effective activation energies $U_{\text {eff }}$ and the attempt times $\tau_{0}$ obtained for each sample from the Arrhenius fits (solid lines) are given. 


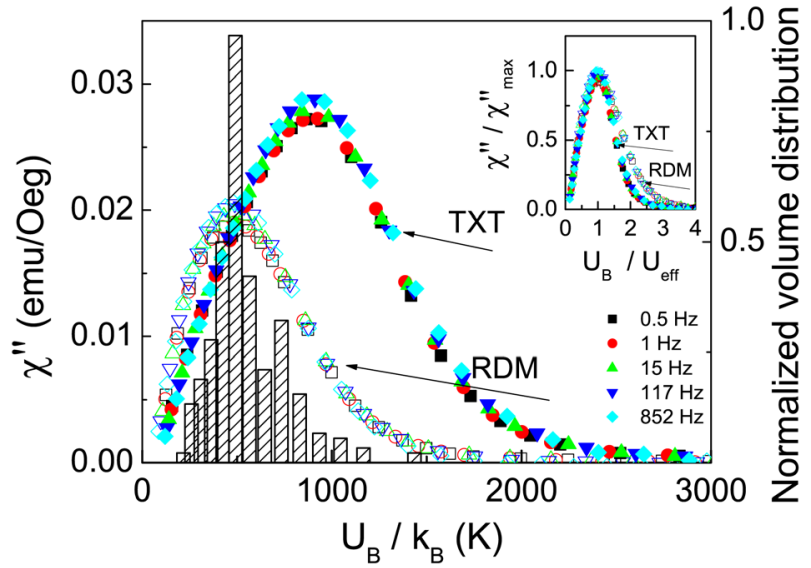

FIG. 8. $\chi^{\prime \prime}$ as a function of the scaling variable $U_{B} / \mathrm{k}_{B}=T \ln \left(1 / \omega \tau_{0}\right)$, with $\tau_{0}=4.64 \times 10^{-12} \mathrm{~s}$ and $\tau_{0}=6.70 \times 10^{-15} \mathrm{~s}$ for RDM and TXT samples, respectively. The size distribution determined by TEM is also included for comparison to the distributions obtained from $\chi^{\prime \prime}$. Inset: $\chi^{\prime \prime}$ scaled to the maxima.

smaller than that of sample RDM. These results confirm that, within the studied range, increasing the average strength of dipolar interactions leads to a much slower magnetic relaxation. This result agrees with the predictions of models, such as those reported in Refs. 8, 12, and 18, which take into account the dynamic fluctuations of dipolar interactions, but it is in contradiction with theories that model such interactions as effective magnetic fields. ${ }^{9,32}$

The distribution of energy barriers $U_{\text {eff }}$ can be obtained by representing the out-of-phase ac susceptibility $\chi^{\prime \prime}$, measured at different frequencies, as a function of the scaling variable $U_{\mathrm{B}} / k_{\mathrm{B}} \equiv T \ln \left(1 / \omega \tau_{0}\right) .{ }^{26}$ Figure 8 shows that the maximum of $\chi^{\prime \prime}$ for TXT sample is shifted towards larger values of $U_{\mathrm{B}}$ with respect to the maximum for the RDM sample. Also, sample TXT shows a narrower $U_{\mathrm{B}}$ distribution. This feature has also been observed in $\gamma-\mathrm{Fe}_{2} \mathrm{O}_{3}$ nanoparticles' assemblies with increasing interaction strength. ${ }^{33}$

\section{CONCLUSIONS}

The application of a magnetic field to a ferrofluid while it is cooled through its freezing temperature induces a magnetic texture through the orientation of the nanoparticles easy axes. This texture is reversible, which means that if the ferrofluid is warmed up to room temperature again, it recovers its initial magnetic properties. After the texturing process, the average strength of dipolar interaction increases and magnetic relaxation becomes slower. This experimental procedure therefore enables a quantitative study of interaction effects while keeping most relevant parameters, such as the distribution of particle sizes, constant.

\section{ACKNOWLEDGMENTS}

This work has been funded by the Spanish MICINN and FEDER, Projects Nos. MAT2007-61621, MAT2009-13977C03 (MOLCHIP), and CONSOLIDER-INGENIO CSD
2007-00010. A.U. and A.A. thank the European Network MAGMANet and the Spanish MICINN (FPI program), respectively, for their scholarships. We acknowledge Labor. Llum Sincrotró for beamtime allocation on BM16-CRG at ESRF in Grenoble. The authors acknowledge F. Fauth for his assistance with the SAXS measurements.

${ }^{1}$ For a recent review, see P. E. Jönsson, J. Nanosci. Nanotechnol. 10, 6067 (2010).

${ }^{2}$ D. J. Sellmyer, M. Yu, and R. D. Kirby, Nanostruct. Mater. 12, 1021 (1999).

${ }^{3}$ E. Chunsheng, D. Smith, J. Wolfe, D. Weller, S. Khizroev, and D. Litvinov, J. Appl. Phys. 98, 024505 (1995).

${ }^{4}$ T. Jonsson, J. Mattson, C. Djuberg, F. A. Khan, P. Nordblad, and P. Svedlindh, Phys. Rev. Lett. 75, 4138 (1995).

${ }^{5}$ C. Djurberg, P. Svedlindh, P. Nordblad, M. F. Hansen, F. Bodker, and S. Mørup, Phys. Rev. Lett. 79, 5154 (1997).

${ }^{6}$ O. Petracic, X. Chen, S. Bedanta, W. Kleemann, S. Sahoo, S. Cardoso, and P. P. Freitas, J. Magn. Magn. Mater. 300, 192 (2006).

${ }^{7}$ R. López-Ruiz, F. Luis, J. Sesé, J. Bartolomé, C. Deranlot, and P. Petroff, EPL 89, 67011 (2010).

${ }^{8}$ J. L. Dormann, L. Bessais, and D. Fiorani, J. Phys. C 21, 2015 (1988).

${ }^{9}$ S. Mørup and E. Tronc, Phys. Rev. Lett. 72, 3278 (1994).

${ }^{10}$ M. F. Hansen and S. Mørup, J. Magn. Magn. Mater. 184, 262 (1998).

${ }^{11}$ J. L. Dormann, D. Fiorani, and E. Tronc, J. Magn. Magn. Mater. 202, 251 (1999).

${ }^{12}$ T. Jonsson, P. Nordblad, and P. Svedlindh, Phys. Rev. B 57, 497 (1998).

${ }^{13}$ W. L. Luo, S. R. Nagel, T. F. Rosenbaum, and R. E. Rosensweig, Phys. Rev. Lett. 67, 2721 (1991).

${ }^{14}$ S. H. Masunaga, R. F. Jardim, R. S. Freitas, and J. Rivas, Appl. Phys. Lett. 98, 013110 (2011).

${ }^{15}$ J. L. Dormann, F. D’Orazio, F. Lucari, E. Tronc, P. Prené, J. P. Jolivet, D. Fiorani, R. Cherkaoui, and M. Noguès, Phys. Rev. B 53, 14291 (1996)

${ }^{16}$ S. Tomita, P. E. Jönsson, K. Akamatsu, H. Nawafune, and H. Takayama, Phys. Rev. B 76, 174432 (2007).

${ }^{17}$ S. Sahoo, O. Petracic, W. Kleemann, P. Nordblad, S. Cardoso, and P. P. Freitas, Phys. Rev. B 67, 214422 (2003).

${ }^{18}$ F. Luis, F. Petroff, J. M. Torres, L. M. García, J. Bartolomé, J. Carrey, and A. Vaurès, Phys. Rev. Lett. 88, 217205 (2002).

${ }^{19}$ J. Wagner, B. Fischer, and T. Autenrieth, J. Chem. Phys. 124, 114901 (2006).

${ }^{20}$ G. Meriguet, F. Cousin, E. Dubois, F. Boue, A. Cebers, B. Farago, and W. Perzynski, J. Phys. Chem. B 110, 4378 (2006).

${ }^{21}$ X. X. Zhang, G. H. Wen, G. Xiao, and S. Sun, J. Magn. Magn. Mater. 261, 21 (2003).

${ }^{22}$ T. Hyeon, S. S. Lee, J. Park, Y. Chung, and H. Bin Na, J. Am. Chem. Soc. 123, 12798 (2001).

${ }^{23}$ D. I. Svergun, J. Appl. Crystallogr. 25, 495 (1992).

${ }^{24}$ K. O'Grady, A. Bradbury, J. Popplewell, S. W. Charles, and R. W. Chantrell, J. Magn. Magn. Mater. 49, 106 (1985).

${ }^{25}$ R. W. Chantrell, N. Y. Ayoub, and J. Popplewell, J. Magn. Magn. Mater. 53, 199 (1985).

${ }^{26}$ F. Luis, J. M. Torres, L. M. García, J. Bartolomé, J. Stankiewicz, F. Petroff, F. Fettar, J. L. Maurice, and A. Vaurès, Phys. Rev. B 65, 094409 (2002).

${ }^{27}$ M. El-Hilo, K. O’Grady, and R. W. Chantrell, J. Magn. Magn. Mater. 117, 21 (1992).

${ }^{28} \mathrm{P}$. Svedlindh, T. Jonsson, and J. L. García-Palacios, J. Magn. Magn. Mater. 169, 323 (1997).

${ }^{29}$ L. Néel, C. R. Acad. Sci. 228, 664 (1949).

${ }^{30}$ W. F. Brown, Phys. Rev. 130, 1677 (1963).

${ }^{31}$ W. T. Coffey, D. S. F. Crothers, Y. P. Kalmykov, E. S. Massawe, and J. T. Waldron, Phys. Rev. E 49, 1869 (1994).

${ }^{32}$ P. E. Jönsson and J. L. García-Palacios, Europhys. Lett. 55, 418 (2001).

${ }^{33}$ J. L. Dormann, D. Fiorani, R. Cherkaoui, E. Tronc, F. Lucari, F. D’Orazio, L. Spinu, M. Noguès, H. Kachkachi, and J. P. Jolivet, J. Magn. Magn. Mater. 203, 23 (1999). 